\title{
Limitations of the Equivalent Core Model For Understanding Core-Level Spectroscopies
}

\author{
Paul S. Bagus, ${ }^{a}$ Carmen Sousa, ${ }^{\mathrm{b}}$ and Francesc Illas ${ }^{\mathrm{b}}$ \\ (Submitted to PCCP - 7/2020) \\ ${ }^{a}$ Department of Chemistry, University of North Texas, Denton, TX 76203-5017, USA \\ ${ }^{b}$ Departament de Ciència de Materials i Química Física \& Institut de Química Teòrica i \\ Computacional (IQTCUB), Universitat de Barcelona, C/Martí i Franquès 1, \\ 08028 Barcelona, Spain
}

\begin{abstract}
.
The equivalent core model, or the $\mathrm{Z}+1$ approximation, has been used to interpret the binding energy, BE, shifts observed in X-ray photoelectron spectroscopy, XPS; in particular to relate these shifts to their origin in the electronic structure of the system. Indeed, a recent paper has claimed that the equivalent core model provides an intuitive chemical view of XPS BE shifts. In the present paper, we present a detailed comparison of the electronic structure provided from rigorous core-hole theory and from the equivalent core model to assess the validity and the utility of the use of the equivalent core model. This comparison shows that the equivalent core model provides a qualitative view of the different properties of initial and core-hole electronic structure. It is also shown that a very serious limitation of the equivalent core model is that it fails to distinguish between initial and final state contributions to the shifts of BEs which seriously reduces the utility of the information obtained with the equivalent core model. Indeed, there is a danger of making an incorrect assignment of the importance of relaxation because the equivalent core model appears to stress the role of final state effects. Given the importance of the distinction of initial and final state effects, we provide rigorous definitions of these two effects and we discuss an example where an incorrect interpretation was made based on the use of the equivalent core model.
\end{abstract}

Keywords: XPS, BE Shifts, Koopmans Theorem, Equivalent Core Model, Initial and Final State Effects 


\section{Introduction}

The equivalent core approximation or model was introduced in the 1970s by Jolly and collaborators [1-3] as a way to understand shifts in the Binding Energies, BEs, observed in X-Ray Photoemission Spectroscopy, XPS. There is a very appealing physical basis for the equivalent core model. The idea is that if one ionizes a deep core electron, the valence electrons see an effective nuclear charge that is larger by essentially unity from that which they saw before the core ionization. Thus, for example, if one removes a 1s electron from $\mathrm{Ne}$, the effective charge seen by the valence, $2 \mathrm{~s}$ and $2 \mathrm{p}$, electrons in increased by one and one can view core ionized $\mathrm{Ne}$ as an $\mathrm{Na}^{+}$cation. The extension to molecular systems is straightforward and much more rewarding in terms of chemical information. Thus, we could view the $\mathrm{CO}$ molecule where a $\mathrm{C}(1 \mathrm{~s})$ electron has been ionized as $\mathrm{NO}^{+}$and where an $\mathrm{O}(1 \mathrm{~s})$ electron has been ionized as $\mathrm{CF}^{+}$. Indeed, Jolly used the equivalent core model of BE shifts to infer thermodynamic properties of various compounds. [1,3] A direct extension can be made for condensed systems. [4-6] Thus, one might consider a core ionized atom in $\mathrm{Ni}$ metal as being a $\mathrm{Cu}$ impurity where, as well as the equivalent core model, we have also used the physical fact that core-holes in systems with equivalent atoms are best viewed as localized on one of these centers; see, for example, Ref. [7]. Johansson and colleagues [4-6] have used the difference of the stability of surface and bulk impurities to interpret the surface core level shifts, SCLS, of the BEs of bulk and surface atoms. Recently, Delesma et al. [8] argued that the equivalent core model provided a way to chemically understand the significance of BE shifts and to use these shifts coupled with calculations of the electronic structure for the equivalent model system to make inferences about the chemical significance of the observed shifts. We agree that the equivalent core model is a useful way to obtain qualitative understanding of the XPS process. It is especially helpful to obtain a chemical view of the extraatomic screening of core-holes; indeed, the different bonding of the original atom and the equivalent core atom is at the heart of the applications made by Jolly and colleagues [1-3] and by Johansson and colleagues. [4-6] The distinction between atomic and extra-atomic screening is especially relevant for understanding the changes in chemistry between the initial state where the core is filled and the final state where there is a core-hole. For example, in Ref. [9] there is an explicit contrast of atomic and extra-atomic relaxation and the differences of these relaxations for different atoms in a compound. However, it is also important to be aware that there are also limitations with the use of the equivalent core model. The principle objective of this paper is to 
provide a sound basis to understand the accuracy and the limitations of the equivalent core model to describe and interpret XPS spectra.

The limitations of the equivalent core model can be divided into several groups: (1) Limitations of the replacement of an atom with a core-hole with the $Z+1$ equivalent ion. (2) Limitations because there are geometric differences between the ionized molecule or compound and the equivalent core system. (3) Neglect of the multiplets that arise from the angular momentum coupling of the open core shell and an open valence shell. This is important because open shell systems are prevalent in chemistry and arise even for simple small molecules and because the multiplet features in XPS provide direct insight into the chemistry and the chemical bonding in a system. [10-11] (4) The angular momentum coupling of the core and valence electrons is also relevant for the states that describe shake satellites. [12] In these states, as well as a core-hole, a valence electron is excited from a filled to an open shell leading to a system with three open shells even if the initial system had been closed shell. Indeed, it is not always possible to rigorously separate shake satellites from the multiplets discussed in (3) above. [13-15] And, (5) Limitations related to the separation and distinguishing of initial and final state contributions to BE shifts. This last may be the most serious of the limitations of the equivalent core model. This is true not because it is a reflection on the accuracy of the equivalent core model but, as we discuss below, because it can lead to incorrect, misleading interpretations of the results of equivalent core model calculations. In the following section, Fundamental Concepts, the basic concepts required to interpret XPS spectra and to understand the equivalent core limitations are reviewed. In the following three sections, specific limitations and their magnitude are analyzed with reference to specific examples. Section III compares the core hole and the equivalent core electronic structure and establishes the magnitude of differences between them. Section IV examines the effects of geometry differences between the original and the equivalent core systems. Section V presents a case study for the separation of initial and final state effects and illustrates the need to use a rigorous determination of these two contributions to BE shifts. Finally, our conclusions are summarized in Section VI. For the most part, we will use HartreeFock, HF, wave functions, WFs, since these WFs are usually adequate to permit a clear and correct understanding of the significance of the XPS in terms of the electronic structure. [11, 1617] However, there will be brief discussion of results obtained with density functional theory, 
DFT, [18-20] and with inclusion of many-body effects in WFs through mixing of configurations or configuration interaction, CI. [21]

\section{Fundamental Concepts}

In this section, rigorous definitions of the important concepts used to analyze XPS are given. These concepts are necessary to obtain the correct interpretation of XPS spectra in terms of the electronic structure of the materials studied.

\section{II.A Initial and Final State Contributions To BEs}

This is a critical distinction which is vital to understand the chemical significance of XPS BEs and, in particular, the physical and chemical meaning of shifts of BEs between atoms in a compound or between atoms in different systems. [11, 16-17] The distinction between initial and final state contributions to BEs, is closely related to what is commonly described as the Koopmans' Theorem, KT, approximation for BEs. [22] However, the distinction and separation of these effects is often made without a rigorous theoretical foundation. Here, we describe the essential physical considerations that permit a rigorous definition of this separation.

A general definition of the $\mathrm{KT} \mathrm{BE}$ is the $\mathrm{BE}$ that is obtained by taking the WF for the $\mathrm{N}-1$ electron ion as

$$
\Psi_{\mathrm{N}-1}{ }^{\mathrm{i}}(\mathrm{KT})=a_{\mathrm{i}} \Psi_{\mathrm{N}}
$$

where $\mathrm{a}$ is an annihilation operator which removes a core electron from the $\mathrm{N}$ electron WF and leaves the orbitals of all other electrons unchanged. The index of the shell from which the electron is removed is denoted as a superscript of $\Psi_{\mathrm{N}-1}$ and a subscript on the annihilation operator $a$. This definition does not require that the WF of Eq.(1) is a single determinant and a HF WF. The physically important feature of this WF is that the only change made to the WF is the removal of an electron from a closed shell orbital with all other aspects of the WF unchanged from their description for the neutral system. This means that the orbitals and the CI mixing coefficients [23] are kept frozen and not allowed to change. In other words, the $\Psi_{\mathrm{N}-1}{ }^{\mathrm{i}}(\mathrm{KT})$ of Eq.(1) will also be described as an FO, frozen orbital, WF to highlight the physical content of the WF; this WF only includes initial state, IS, effects since relaxation in response to the core-hole is explicitly excluded. The KT BE is then defined as 


$$
\mathrm{BE}_{\mathrm{i}}(\mathrm{KT})=<\Psi_{\mathrm{N}-1}{ }^{\mathrm{i}}(\mathrm{KT})|\mathrm{H}| \Psi_{\mathrm{N}-1}{ }^{\mathrm{i}}(\mathrm{KT})>-<\Psi_{\mathrm{N}}|\mathrm{H}| \Psi_{\mathrm{N}}>.
$$

When Eqs. (1) and (2) are applied to closed shell HF WFs then $\mathrm{BE}_{\mathrm{i}}(\mathrm{KT})=-\varepsilon_{\mathrm{i}}$, where $\varepsilon_{\mathrm{i}}$ is the HF orbital energy and this relationship is often taken as the meaning of KT. The original use of KT was to obtain approximations to BEs without the need for calculation of the WF of the N-1 electron system. Indeed for the removal of the most weakly bound electron, the first IP, the KT BE is a reasonably good approximation to the IP. [24] It is, however, a very poor approximation to the BEs of core levels and is typically too large by several $10 \mathrm{~s} \mathrm{of} \mathrm{eV} \mathrm{[16]} \mathrm{because} \mathrm{it} \mathrm{neglects}$ the relaxation of the valence orbitals due to the presence of the core-hole; i.e., the screening of the core-hole. The relations of Eqs. (1) and (2) are quite general and can be applied to correlated CI WFs as well as HF WFs; the only constraint is that the shell to be ionized must not be correlated and must be fully occupied in all the configurations of the CI expansion. [23] It is also possible to generalize the relations of Eqs.(1) and (2) to open shell systems taking into account the angular momentum coupling of the open core shell and the open valence shell. [10, 25] Furthermore, these relations can also be rigorously generalized to be applied to DFT densities. [26-27] Since, these generalized KT BEs do not include relaxation, it is preferable to describe them as frozen orbital, FO, BEs. Thus, the shifts of the FO BEs between different atoms of a given element in the system or between the element in different systems are, to an extremely good approximation, the change in the potential of the valence electrons at the different sites in the neutral, unionized, system. This is a chemically important distinction since it allows extracting information of the electronic structure of the systems before ionization takes place. It is important to point out that the potential the valence electrons generate at the nuclei in the neutral system may change because: (1) there is a charge transfer from or to the atom that is ionized; or, (2) there is a different environmental charge density around that atom; or, (3) there is a change in the hybridization of that atom. See, for example Refs. [11, 28-30]. While the initial state or FO BE shifts are not observable, it is an important role of theory to identify these effects and to distinguish them from the final state or relaxation effects to ensure that one extracts information from XPS about the neutral system rather than about the core-ionized system. Furthermore, the theory can identify the electronic structure effects responsible for changes in the initial state potential.[28]

The relaxation effects are included by making a second variational calculation for a configuration that explicitly contains the core-hole and, hence includes the effects of orbital 
relaxation. This variational calculation of the ionic state leads to a WF denoted $\Psi_{\mathrm{N}-1}{ }^{\mathrm{i}}(\mathrm{SCF})$ and the $\mathrm{BE}$ is the difference of the variational energies of the initial $\mathrm{N}$ electron $\mathrm{WF}, \Psi_{\mathrm{N}}$, and $\Psi_{\mathrm{N}-1}{ }^{\mathrm{i}}(\mathrm{SCF})$; it is described as a $\Delta \mathrm{SCF} \mathrm{BE}$ or $\mathrm{BE}(\triangle \mathrm{SCF})$; this definition of $\mathrm{BE}(\Delta \mathrm{SCF})$ can be directly extended to DFT energies. In general, there may be several ionic states for ionization of an electron from the $i^{\text {th }}$ core shell. These states may arise from the angular coupling of the open core and valence shell electrons and they may arise from many body effects as described in the following two sub-sections. In general, when there is a concern to separate initial and final state contributions to BEs, this is done for the most intense, usually the leading, XPS peak which is often described as the "main" XPS peak. The $\mathrm{BE}(\triangle \mathrm{SCF})$ for this main peak state includes the effects of both the initial state potential and the final state relaxation; for an extended description of the relaxation energies and the $\triangle \mathrm{SCF}$ procedure, see, for example, Ref. [16]. It is known that $\mathrm{HF} \mathrm{BE}(\triangle \mathrm{SCF})$ give reasonably accurate core level BEs. [31] It is also known that DFT, using a suitable functional may provide even higher accuracy for core-level BEs. [26-27] The relevance of this discussion for the BE shifts obtained with the equivalent core model is that these BEs do not distinguish initial and final state effects but provide estimates of the $\triangle \mathrm{SCF} B E$ shifts. This is true both for equivalent core model estimates from thermodynamic considerations including using a Born-Haber cycle [1-2, 4-6] or from rigorous calculations of the electronic structure of equivalent core models of a molecule or a condensed phase system. [8] It is also important to stress that with the equivalent core model one cannot obtain BE shifts between the BEs for atoms with different nuclear charges. One can only obtain the shifts of BEs, $\triangle B E s$, between inequivalent atoms with the same nuclear charge. As part of the discussion of the accuracy of the equivalent core model in Sec. III, we point out that the equivalent core model, as well as not being able to provide absolute $\mathrm{BEs}$, also cannot provide a meaningful value for the $\Delta \mathrm{BE}$ between the $\mathrm{O}(1 \mathrm{~s})$ and $\mathrm{C}(1 \mathrm{~s}) \mathrm{BEs}$ in $\mathrm{CO}$.

\section{II.B Multiplets}

Multiplets are critically important for the XPS of open shell systems. The coupling of spin and orbital angular momentum between the ionized core shell and the open valence shell leads to final states with different energies and intensities. An early attempt to treat this angular momentum coupling to describe the cation $2 p$ XPS of transition metal ionic systems that are high spin was carried out by Gupta and Sen. [32-33] This early work was based on using the angular 
momentum coupling of atomic orbitals to predict the XPS spectra of compounds. More recently, the treatment of the multiplets for the $2 p$ XPS of transition metal oxides was carried out based on rigorous WF treatments of cluster models; see, for example, Refs. [11, 15, 34]. These cluster model treatments showed that the multiplet splittings obtained for the atoms are modified by the covalent bonding in the compounds since these covalent interactions change the exchange integrals between the open shell core and valence orbitals. These splittings can and do broaden an observed XPS peak, by a few eV, because of unresolved or partially resolved multiplets. A generalization of the multiplet splittings to include atomic and intra-atomic many-body effects is also often described as a multiplet splitting even though it goes beyond the angular momentum coupling of the open shell electrons. [14, 35-36] Multiplet splitting in XPS is very common and arises even for simple molecules such as $\mathrm{NO}$ and $\mathrm{O}_{2}$ where it gives insight into the electronic structure of the molecules. [10-11] Typically, the energy separations of these multiplets are treated with CI wavefunctions. $[11,16]$ In the normal usage of the equivalent core model, based either on thermodynamic data, [1-3] or on the calculation of the electronic structure of equivalent core models, [8] the multiplet splittings are neglected and an important part of the XPS spectra, especially of open shell systems, is neglected. Indeed, the position of the core-hole needs to be taken into account since the magnitude of these splittings strongly depend on the core-level ionized. [37-38] Clearly, for information about multiplet splittings, crucial for understanding the XPS of open shell systems, there does not seem to be new value or insight from the equivalent core model.

\section{II.C XPS Features and Satellites}

In order to properly understand the significance of features of the XPS spectra for the electronic structure and bonding of a system, it is very helpful to be able to predict or extract information from the number and the intensities of the features in XPS spectra. There are several origins of the complex features in an XPS spectrum. These include the multiplets that we have discussed above. However, there are many electron effects that involve the core-hole and lead to major changes in intensity; see, for example Refs. [39-41] and there are also features described as shake satellites. [12, 16, 42] For the many electron effects that involve the core level, one redistributes the electrons in the shell that contains the core ion as well as a valence open shell, typically the $n=3$ shell of $3 d$ transition metal complexes, to form configurations. These configurations, while XPS forbidden in themselves mix with the XPS allowed configurations and 
steal intensity leading to a spectrum much more complex than would arise even from consideration of angular momentum coupling; see, for example, Ref. [16]. Clearly, these features, albeit specialized to transition metal, lanthanide, and actinide compounds [16] and metals, [43] cannot be treated with the equivalent core model for the same reason discussed in detail for multiplets in Sec. II.B. On the other hand, shake satellites, which are quite general can and have been treated with the equivalent core model with both DFT (for example, Refs.[44-47]) and semiempirical formalisms (for example Refs.[48-49]). For semi-empirical methods, e.g., INDO [49] or PPP [48], it is necessary to use the equivalent core model since the core electrons are not included in these approximations. A contributing reason for using the equivalent core model with DFT may be that certain standardly used software packages do not allow making a core-level an open shell and the equivalent core approximation must be used for such excited states; see, for example, Refs. [50-51]. We consider a potential limitation of the equivalent core model for the treatment of XPS shake satellites.

Shake satellites involve electron configurations where a core electron is ionized and a valence electron is promoted into an unoccupied spin-orbital. These shake satellites carry considerable intensity although it may be distributed over many different final states; see, for example, Refs. [12, 38, 52]. The intensity of these satellites is calculated with the sudden approximation, SA, [12, 52] see also Ref. [16]. The intensity in the SA is an overlap integral between the initial WF where a core electron has been removed, $\Psi_{\mathrm{N}-1}{ }^{\mathrm{i}}(\mathrm{KT})$ of Eq.(1), and the core-ionized WF for the shake excitations. This overlap can easily be calculated with equivalent core model WFs with the constraint that the angular momentum coupling of the core open shell with the valence open shells cannot not be included. The valence open shells for a shake excitation in, for example, $\mathrm{C}_{6} \mathrm{H}_{6}$ would have the configuration $1 \mathrm{e}^{3} 2 \mathrm{e}^{1}$ where the main hole configuration is $1 \mathrm{e}^{4}$; the principle quantum numbers $1 \mathrm{e}$ and $2 \mathrm{e}$ are arbitrary. These two open shells can couple to either singlet $(\mathrm{S})$ or triplet $(\mathrm{T}) ;[45,53]$ the selection rules are such that only the singlet coupling can get intensity. [45] However in an all electron WF where the open core shell is present, the open core shell electron can recouple with the open valence shell electron to give a total doublet. When this coupling is taken into account, the XPS allowed triplet valence shell coupling and the XPS forbidden singlet valence shell coupling can mix and both shake states will have intensity. This is relevant for the shake spectra of $\mathrm{C}_{6} \mathrm{H}_{6}$ since there is both experimental [54] and theoretical [53] evidence that the nominally forbidden $\mathrm{T}$ coupled $1 \mathrm{e}^{3} 2 \mathrm{e}^{1}$ 
configuration can gain significant intensity and cannot be neglected. There is also evidence that the coupling first of valence shells to $\mathrm{S}$ and $\mathrm{T}$ and then coupling with the core level to the total doublet multiplicity may not be the best order of angular momentum coupling. For the shake spectra of the Ne 1s XPS, Martensson et al. [55] have chosen to couple the $2 p$ with the $1 \mathrm{~s}$ first to $\mathrm{S}$ and $\mathrm{T}$ and then couple to the excited $\mathrm{p}$ orbital rather than to couple the $2 \mathrm{p}$ with the excited $\mathrm{p}$ orbital first to $\mathrm{S}$ and $\mathrm{T}$. They found that the coupling of the $1 \mathrm{~s}$ with $2 \mathrm{p}$ first was physically correct and allowed them to explain the two series that are observed as XPS shake satellites in the Ne 1s XPS. These considerations suggest that the equivalent model should be used with caution in attempting to assign XPS shake satellites.

\section{Accuracy of The Equivalent Core Model Orbitals}

Delesma et al. [8] have made a strong case that the numerical accuracy of BE energy shifts obtained for representative cases is reasonably high, with errors of order $0.1 \mathrm{eV}$ or less. It is, however, worthwhile to directly examine the properties of the orbitals themselves to identify the departure of the character of the orbitals of equivalent core models from the orbitals of configurations where the core-hole is explicitly treated. This is done for the representative molecule $\mathrm{CO}$ where we contrast the properties of the ground state of $\mathrm{CO}$ with the $\mathrm{C}(1 \mathrm{~s})$ ion, $\mathrm{C}(1 \mathrm{~s})-\mathrm{CO}^{+}$, and the $\mathrm{O}(1 \mathrm{~s})$ ion, $\mathrm{O}(1 \mathrm{~s})-\mathrm{CO}^{+}$. For this purpose, we use Complete Active Space, CAS, [56] WFs and orbitals where the many body effects are treated by distributing the $4 \pi$ electrons in all ways over the nearly degenerate $1 \pi$ and $2 \pi$ orbitals. Our objective with these calculations is to compare the properties of rigorous, all electron, WFs for the core-hole configurations of $\mathrm{CO}$ with comparable equivalent core model WFs for $\mathrm{NO}^{+}$and $\mathrm{CF}^{+}$. We use CAS WFs to avoid the artifacts of SCF calculations for the BEs that lead to an anomalous sign of the error of the $\mathrm{C}(1 \mathrm{~s}) \mathrm{BE}$. The basis sets for all the CAS WFs are extended and they are uncontracted to ensure that our BE calculations will be accurate. Further details of the CAS calculations are given in Ref. [23]. The CAS case is chosen because it contains the important static correlation effects, see, for example, Refs. [57-58], that lead to accurate core level BEs for CO [23] when they are computed as $\triangle$ SCF BEs. Since the XPS ionization is a vertical process, [16] all BEs are for the equilibrium $\mathrm{R}(\mathrm{C}-\mathrm{O})=1.128 \AA$. [59] The CAS BEs are for non-relativistic WFs and a correction for relativistic effects, as found for the 1s ionization of isolated $\mathrm{C}$ and $\mathrm{O}$ atoms, see Ref. [23], is also included in Table I. The accuracy of the BEs is demonstrated in 
Table I where the calculated BEs are compared to experiment. [60] As well as the individual $\mathrm{C}(1 \mathrm{~s})$ and $\mathrm{O}(1 \mathrm{~s}) \mathrm{BEs}$, we also give the $\mathrm{BE}$ shift between the $\mathrm{O}(1 \mathrm{~s})$ and $\mathrm{C}(1 \mathrm{~s}) \mathrm{BEs} ; \Delta \mathrm{BE}=$ $\mathrm{BE}[\mathrm{O}(1 \mathrm{~s})]-\mathrm{BE}[\mathrm{C}(1 \mathrm{~s})]$. Once the relativistic effect is included, the $\mathrm{CAS} \mathrm{C}(1 \mathrm{~s})$ and $\mathrm{O}(1 \mathrm{~s}) \mathrm{BEs}$ are, as expected, smaller than experiment. However, the critical fact is that they are both smaller by the same amount, $0.3 \mathrm{eV}$. This leads to a theoretical value for $\Delta \mathrm{BE}$ that is in agreement with experiment. In order to further reduce the $0.3 \mathrm{eV}$ error in the absolute value of the BEs, additional dynamic correlation effects [57-58] will have to be included. Since the accuracy of the BEs shown in Table I is sufficient to insure that comparison, given below, of the properties of the rigorous WFs where the core electrons are included with the equivalent core model WFs is reliable. Furthermore, we point out that the equivalent core model cannot be used to determine the $\mathrm{BE}$ shift from $\mathrm{C}(1 \mathrm{~s})$ to $\mathrm{O}(1 \mathrm{~s})$ since the total energies depend strongly on the elemental composition of the equivalent molecules. The difference of the total energies of the equivalent molecules, $\mathrm{CF}^{+}$and $\mathrm{NO}^{+}$, is $215 \mathrm{eV}$, over $30 \mathrm{eV}$ different from the correct value of $\Delta \mathrm{BE}$. However, the agreement of the calculated BEs and $\triangle \mathrm{BE}$ with experiment is a strong basis for confidence that the CAS WFs accurately describe the electronic structure and allow us to compare and contrast the orbital and WF properties of the hole-state WFs with the equivalent core WFs.

In the present work, we have extended the CAS calculations for $\mathrm{CO}$ in Ref. [23] to comparable CAS calculations for the equivalent core model molecules $\mathrm{NO}^{+}$and $\mathrm{CF}^{+}$. The comparison of the $\mathrm{NO}^{+}$with $\mathrm{C}(1 \mathrm{~s})-\mathrm{CO}^{+}$and the $\mathrm{CF}^{+}$with $\mathrm{O}(1 \mathrm{~s})-\mathrm{CO}^{+} \mathrm{CAS}$ WFs will provide detailed information on how similar or different the equivalent core WFs are from the rigorous hole configuration WFs. The CAS WFs for the equivalent core molecules include the same many-body effects and are at the same internuclear distance as for CO; thus, they are directly comparable. In Table II, we compare the occupation numbers of the $1 \pi$ and $2 \pi$ orbitals, $N(1 \pi)$ and $N(2 \pi)$, for the ground state of CO, the rigorous core-ions, and the equivalent core CAS WFs. For HF WFs, the occupation numbers are $N(1 \pi)=4$ and $N(2 \pi)=0$. The departure of $\mathrm{N}(2 \pi)$ from zero indicates the importance of the static many-body effects that are needed to accurately describe the $C(1 \mathrm{~s})$ and $\mathrm{O}(1 \mathrm{~s})$ BEs. [23] We consider first the

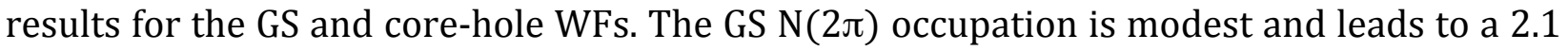
eV lowering of the GS energy. [23] The occupation of the $2 \pi$ orbital is larger for the $\mathrm{C}(1 \mathrm{~s})$ 
ion and smaller for the $\mathrm{O}(1 \mathrm{~s})$ ion and the energy lowerings of the CAS core-hole over HF WFs follow the same trend. Indeed, it is this differential energy lowering that leads to a correct description of 1s BEs. In our earlier work, [23] the difference in the importance of many-body effects was assigned to the degree of charge separation, largest when the atomic charges were similar and smaller when the atomic charges were more different. The same pattern of the $\mathrm{N}(2 \pi)$ occupation numbers is found for the equivalent core molecules where the $\mathrm{N}(2 \pi)$ is larger for $\mathrm{NO}^{+}$than for $\mathrm{CF}^{+}$. However, the extent of the static many body effects is smaller for the equivalent core molecules than for the molecules with a rigorous corehole; for the equivalent molecules, the $\mathrm{N}(2 \pi)$ are only $90 \%$ of the values for the core-hole molecules. This shows that the valence orbitals of the equivalent core molecules, while reproducing some of the important features, are not quite the same as those of the corehole molecules.

The centers of charge of the valence orbitals for the various CAS WFs is examined in Table III. As for Table II, values for the core-hole ions are from Ref. [23] and the equivalent core model systems, denoted EQC in the table, are from the present work. The atoms are on the $\mathrm{z}$ axis and the centers of charge, or expectation values of $\mathrm{z},\langle\mathrm{z}\rangle$, are taken with respect to midpoint of the molecule where the $\mathrm{C}$ (or $\mathrm{N}$ ) nucleus is at $\mathrm{z}=-0.564 \AA$ and the $\mathrm{O}$ (or F) nucleus is $\mathrm{z}=+0.564 \AA$. Positive values of $\langle\mathrm{z}>$ indicate an orbital polarized toward $\mathrm{O}$ (or F) and $<\mathrm{z}><0$ indicates an orbital polarized toward $\mathrm{C}$ ( or $\mathrm{N}$ ). Large magnitudes of $<\mathrm{z}>$, comparable to or greater than $0.5 \AA$, indicate an orbital with considerable lone pair character. The center of charge of the 6 valence $\sigma$ electrons, $3 \sigma^{2} 4 \sigma^{2} 5 \sigma^{2}$, is also given as $\operatorname{sum}(\sigma)$. Since these orbitals are doubly occupied, any unitary transformation of the orbitals yields identical WFs and only sum( $\sigma)$ is invariant. The $\pi$ orbitals are the natural orbitals of the CAS WFs $[56,61]$ and are the orbitals associated with the occupation numbers in Table II. For the CO GS, the $<z>$ of the valence $\sigma$ orbitals show that the $3 \sigma$ orbital has its center of charge near the center of the $\mathrm{CO}$, the $4 \sigma$ is a lone pair with its center of charge slightly outside of 0 , and the $5 \sigma$ is a lone pair with a center of charge considerably outside of $\mathrm{C}$. The center of charge of the six $\sigma$ electrons is nearly at the center of the molecule. For the corehole WFs, the $\sigma$ orbitals have a reasonably similar character. For the $\mathrm{O}(1 \mathrm{~s})-\mathrm{CO}^{+} \mathrm{WF}$, the $\sigma$ electron charge is polarized toward the 0 atom where the electron has been removed. For 
the CO GS, the $1 \pi$ is a bonding orbital with a center of charge about $3 / 4$ of the distance between C and $\mathrm{O}$, toward $\mathrm{O}$; i.e., dominantly on $\mathrm{O}$ while the $2 \pi$, which has a small occupation is antibonding and roughly midway between $\mathrm{C}$ and $\mathrm{O}$. For the $\mathrm{C}(1 \mathrm{~s})$ ion, the bonding $1 \pi$ shifts its center of charge toward $\mathrm{C}$ as would be expected for the equivalent core molecule $\mathrm{NO}^{+}$while for the $\mathrm{O}(1 \mathrm{~s})$, the $<\mathrm{z}>$ of the bonding $1 \pi$ shifts much closer toward the $\mathrm{O}$ center again as would be expected for $\mathrm{CF}^{+}$. The trends of the centers of charge of the valence orbitals for the core ions are also found for the equivalent core molecules but they are not reproduced perfectly as may be seen from the ratios of the $<\mathrm{z}>$ between the equivalent core molecules and the rigorous core ions.

The overall evidence presented in this section shows that while the equivalent core model provides a qualitative view of the screening of a core hole by replacing the ionized atom with the

next atom in the periodic table, there are quantitative limitations. This is not surprising if one considers that the screening of the nuclear charge by a deep, 1s, orbital is not precisely one. Slater developed rules to estimate the screening of the nuclear charge by the core and valence electrons in an atom. In particular for first row atoms, as relevant for our test system of CO, Slater concluded that the screening of the nuclear charge seen by the valence electrons by a 1 s electron is 0.85 rather than 1. [62]

\section{Geometric Differences}

The equilibrium bond distances for core-ionized systems are different from those for the initial state of a system before ionization. Delesma et al. [8] have pointed out that for the C(1s) ionization of $\mathrm{CO}$ the $\mathrm{C}-\mathrm{O}$ bond distance is reduced by $0.05 \AA$. Changes in bond distances can and do occur for valence ionizations as well as for core ionization; for example, the changes in bond distance are particularly important for the ionization of the deepest valence level of $\mathrm{CO}$, the bonding $3 \sigma$ orbital. [63] In addition changes in bond length upon core ionization are also found for the ionic compounds as well as for simple molecules. For $\mathrm{MgO}$, for example, the bond distance from $\mathrm{Mg}$ to $\mathrm{O}$ is reduced by $0.12 \AA$ when an $\mathrm{Mg} 2 \mathrm{p}$ electron is ionized. [64] However, since XPS is a vertical process, the bond-length changes in the ionic states lead to vibrational excitations which are not always resolved; see, for example, Refs. [63-67]. When the vibrational excitations are not resolved, the Franck-Condon broadening can make a significant contribution to the observed XPS, $\sim 2 \mathrm{eV}$ for the $3 \sigma$ XPS of CO [63] and $\sim 1 \mathrm{eV}$ for the Mg 2p XPS of MgO. 
[64] Of course, the observed broadening will be a convolution of the vibrational broadening with other broadening, especially arising from experimental resolution. [64]

Equivalent core molecules have different bond lengths than the ground states of the ionized molecule and this difference may be relevant for the BE shifts deduced from the equivalent core model. The bond lengths for the equivalent system are closer to the bond lengths of the core ionized system which, as discussed above, may be quite different from the unionized system. However, since XPS is a vertical process, the geometry changes in the ionized system will mainly lead to Franck-Condon broadenings of a peak whose center is close to the vertical ionization with the ground state geometry. For theoretical calculations of the equivalent core molecules, this is not a problem since the geometry can be fixed as in the initial state. [8] However, for shifts deduced from thermodynamic data for the equivalent core systems, [1-3, 5-6] this may be a problem since the thermodynamic data refers to the geometry of the equivalent core systems not to the geometry of the system whose XPS is studied. In order to determine the connection between changes in the molecular geometry and changes in the BEs, we have extended the CAS CO WFs in Ref. [23] to examine changes in $d(C-O)$ about $r_{e}(C O)$. [59] The absolute BEs for $r_{e}$ are given in Table I; in Table IV, we report changes in these BEs, $\delta \mathrm{BE}[\mathrm{d}, \mathrm{C}(1 \mathrm{~s})]$ and $\delta \mathrm{BE}[\mathrm{d}, \mathrm{O}(1 \mathrm{~s})]$, for changes in $\mathrm{d}(\mathrm{C}-\mathrm{O})$ about $\mathrm{r}_{\mathrm{e}}$ in the range $\sim \pm 0.1 \AA$. The changes are larger for the $\mathrm{C}(1 \mathrm{~s})$ BEs than for the $\mathrm{O}(1 \mathrm{~s})$ BEs. The implication of the data in Table $\mathrm{V}$ for the use of the equivalent core model is that the geometries appropriate for the equivalent system may lead to uncertainties in the BE shifts of order a few tenths of an $\mathrm{eV}$.

\section{Interpretation of Equivalent Core BE Shifts}

Here we address a concern not so much for the nature of the equivalent core approximation but for the interpretation of the BE shifts obtained with the equivalent core model as reflecting the screening or relaxation in response to the core hole. As we pointed out in the discussion in Sec. II.A, the BE shifts obtained with the use of the equivalent core model combine both initial state and final state contributions. While it is tempting to assign the shifts obtained with the equivalent core model to final state effects, or core-hole screening, this may be misleading and it is necessary to use a formalism where initial state and final state contributions can be rigorously separated. This is quite important because while initial state effects can be 
directly related to the electronic structure of the unionized system; the same direct relationship is not possible for the final state contributions. [11, 68] In other words, even if it may seem a philosophical issue, the distinction between initial and final state effects is crucial, especially if one is to obtain physically and chemically meaningful conclusions from the XPS data.As an example of the dangers of such a mis-assignment of BE shifts to final state effects, we consider the shifts of BEs between atoms in the bulk of a solid and those at the surface which are described as surface core level shifts, SCLS; see for example Refs. [11, 16, 69].

In order to illustrate the power of using the equivalent core model, Delesma et al. [8] consider the SCLS along the $4 \mathrm{~d}$ transition series of metals from Y to Cd. The SCLS is defined such that for SCLS $>0$ the surface BE is larger than the bulk BE. Their calculations show a trend for positive SCLS at the left of the row to negative SCLS, or bulk BE being larger than the surface BE, at the right end of the row. This trend along a transition metal atom row is consistent with measurements, for example, for the $5 \mathrm{~d}$ transition metal elements. [69] The origin of this trend is interpreted by Delesma et al. [8] in terms of the screening of the core-level ion at bulk and surface and it is argued that the screening is more effective for the bulk when the $\mathrm{d}$ shell is less than half filled leading to positive SCLS at the left of the row. In other words, the trend predicted from the equivalent core model calculations is described as a final state, relaxation or screening, effect. However this interpretation is not supported by a rigorous separation of initial and final state contributions, defined in Sec. II.A, to the SCLS. Indeed, this separation is not possible if only WFs or DFT densities for the equivalent core model are used since the BE shifts obtained with this model include both initial and final state contributions; see Sec. II.A. From extensive earlier work on a number of different systems [9, 11, 16, 70-73] ranging from metals to oxides, where there has been a rigorous separation of initial and final state contributions, a quite different conclusion is reached. In Table V, the SCLS are presented using the notation defined in Sec. II.A, BE(KT) for initial state BEs and BE $(\triangle \mathrm{SCF})$ for BEs that include both initial and final state contributions. The data for the systems in Table $\mathrm{V}$ proves that the initial state, or KT, contributions dominate to determine the magnitude and sign of the SCLS. The specific initial state effects that are responsible for the SCSL are described in the original papers.

For the results in Table V, the SCLS obtained with the BE(KT) are denoted SCLS(KT) and the SCLS obtained with the $\mathrm{BE}(\triangle \mathrm{SCF})$ are denoted SCLS $(\triangle \mathrm{SCF})$. The experimental results for the SCLS are denoted SCLS(expt). Before we turn to an analysis of the decomposition of the 
SCLS into initial and final state contributions, we briefly describe the theoretical models used to obtain bulk and surface BEs to indicate how the distinction of bulk and surface BEs is made; details are provided in the original papers. To model the SCLS of fcc $\mathrm{Al}(100)$ and $\mathrm{Cu}(100)$, [74] 18 atom clusters with 4 layers, written as $\mathrm{X}_{5} \mathrm{X}_{4} \mathrm{X}_{5} \mathrm{X}_{4}$ with $\mathrm{X}=\mathrm{Al}$ and $\mathrm{Cu}$, were used. [72-73] These clusters contain a representative surface atom and a representative bulk atom at the centers of the first and third $\mathrm{X}_{5}$ layers, respectively. Initial and final, core-hole, HF WFs are used to compute the surface and bulk $\mathrm{BE}(\mathrm{KT})$ and $\mathrm{BE}(\Delta \mathrm{SCF})$. For the insulating $\mathrm{MgO}[71]$ and $\mathrm{CaO}[9$, 70] oxides, large clusters with Evjen [75] point charge embedding to insure that the Madelung potential is correctly distinguished between surface and bulk were used. Several cluster sizes were used with similar results for the SCLS but only results for the largest are reported in Table V. For each oxide four clusters were used, two to describe the surface and bulk atoms of the metal cation and two to describe the surface and bulk atoms of the $\mathrm{O}$ anion. Clusters for the $\mathrm{O}$ SCLS of $\mathrm{CaO}$, which involved four shells of atoms with a central $\mathrm{O}$ atom, were $\mathrm{OCa}_{6} \mathrm{O}_{18} \mathrm{Ca}_{38}$ for the bulk and $\mathrm{OCa}_{5} \mathrm{O}_{13} \mathrm{Ca}_{25}$ for the surface; similar size clusters were used for the other $\mathrm{CaO}$ and MgO SCLS BEs. Initial and final HF WFs were used to obtain the SCLS for the O(1s). $\operatorname{Mg}(2 \mathrm{p})$, and $\mathrm{Ca}(2 \mathrm{p})$ BEs. To prove that the cluster models properly represent the physics that distinguishes the bulk and surface atom BEs, slab model DFT densities were also used to determine the SCLS. [70] The slabs of $\mathrm{CaO}(100)$ had 5 layers where the central layer was taken to represent the bulk; a PBE functional was used. In Table $\mathrm{V}$, the $\mathrm{CaO}$ cluster results for the SCLS are labelled $\mathrm{CaO}(100)$-cluster to be contrasted with the slab SCLS labelled CaO(100)-slab. The purpose is to compare the initial state and final state contributions to the SCLS and, where possible, to compare with experiment.

For $\mathrm{Cu}(100)$, the SCLS(KT) for the deepest 1s orbital has the same magnitude and sign as the $\operatorname{SCLS}(\triangle \mathrm{SCF})$ which includes final state relaxation, although the SCLS $\triangle \mathrm{SCF})$ is $0.25 \mathrm{eV}$ smaller in magnitude. The $\mathrm{Cu}$ 1s SCLS also has the same sign and magnitude as the measured $\mathrm{Cu}$ $2 p$ SCLS. [73] From the results for the Al(100) SCLS, to be discussed next, the Cu 2p SCLS is expected to be similar, albeit somewhat smaller in magnitude, than the $\mathrm{Cu}$ 1s SCLS. For $\mathrm{Al}(100)$, results for the $1 \mathrm{~s}, 2 \mathrm{~s}$, and $2 \mathrm{p}$ SCLS are shown. The SCLS(KT) is similar for all levels. The $\operatorname{SCLS}(\triangle \mathrm{SCF})$, given only for the Al 1s orbital, is similar to the SCLS(KT). The Al 2p SCLS(KT) has the same sign as experiment and both are reasonably small and positive. The different signs of the SCLS(expt) for $\mathrm{Cu}$ and $\mathrm{Al}$ are reproduced by the theory and, most important, the theory 
shows that this difference is sign is an initial state, SCLS(KT), effect and does not arise from a difference in the screening of $\mathrm{Al}$ and $\mathrm{Cu}$ core holes. It is clear that the initial state differences in the bonding and environment of bulk and surface atoms are responsible for the SCLS of Cu and $\mathrm{Al}$; the relaxation only modifies the absolute value of the SCLS. Thus, while the screening of the core-holes is quite large, [16] it is the same, within $0.25 \mathrm{eV}$, for bulk and surface atoms. The reason for the small SCLS is, as shown in the original papers, due to the cancellation of two initial state contributions one of which leads to a modestly large $\mathrm{SCLS}<0$ while the other leads to an SCLS $>0$. The SCLS, including the different signs for $\mathrm{Cu}$ and $\mathrm{Al}$, are not due to a final state screening as inferred by Delesma et al. [8] from their equivalent core model calculations. The importance of the initial state contributions to the SCLS also holds for the ionic insulators $\mathrm{MgO}$ and $\mathrm{CaO}$ where the SCLS is quite different between core ionization of the metal cation and of the $\mathrm{O}$ anion. For the $\mathrm{Mg} 2 \mathrm{p}$ BEs in $\mathrm{MgO}$ and the $\mathrm{Ca} 2 \mathrm{p}$ BEs in $\mathrm{CaO}$, the $\mathrm{KT}, \Delta \mathrm{SCF}$ and experimental SCLS are reasonably similar and the SCLS arises mostly from initial state effects. The initial state effects for the cation $2 p$ SCLS depend largely on the change in the Madelung potential at a surface atom to a larger, less negative, value compared to the potential at a bulk atom. [71] For the SCLS of the $\mathrm{O}(1 \mathrm{~s}) \mathrm{BEs}$ of $\mathrm{MgO}$ and $\mathrm{CaO}$, initial state contributions to the SCLS also dominate. The $\mathrm{O}(1 \mathrm{~s}) \mathrm{SCLS}$ is calculated to be small and this is consistent with the fact that the $\mathrm{O}(1 \mathrm{~s})$ SCLS is not resolved in the XPS measurements. [71] Based on this knowledge of the importance of initial state effects for the very different cation and O(1s) SCLS, it was possible to identify the surface chemistry which was responsible for the different SCLS [71] of cation and ligand. The results for the $\mathrm{CaO}$ SCLS closely parallel the MgO SCLS especially for the importance of initial state effects, as opposed to final state screening [9, 70] demonstrating the generality of the mechanisms responsible for the SCLS of these two oxides. For CaO, the SCLS obtained with periodic DFT slab models for $\mathrm{CaO}(100)$ [70] are similar to the HF cluster model SCLS, especially as concerns: (1) the large difference of the cation and anion SCLS and (2) the importance of initial state effects. The SCLS from DFT cluster models of $\mathrm{CaO}(100)$, [9, 70] not shown in Table V, are fully consistent with the HF cluster and DFT slab model SCLS shown in the table. Thus, the key chemistry and physics obtained by separating initial and final state effects, directly possible with our core-hole WFs and densities are the same with all three approximations, powerful evidence that our conclusions are correct. Since the importance of initial state effects for the SCLS is shown for these very different cases, it is reasonable to conclude that importance of these effects is general. 


\section{Conclusions}

A detailed analysis and comparison of rigorous core-hole electronic structure with the electronic structure obtained with the equivalent core model has been presented. This comparison has made it possible to understand and place in context both the advantages of the equivalent core model and the dangers and limitations of an uncritical use of the model. The model provides a useful qualitative and graphical guide to the consequences of the relaxation and response to coreholes. We have shown that there are differences in the character of the valence orbitals between the rigorous core-hole WFs and the equivalent core model WFs but they are relatively minor. In particular, the trends over different hole states obtained with rigorous core-hole WFs and equivalent core WFs for orbital character and for many-body effects are similar. One of the novel and unexpected things that we have shown concerns the use of equivalent core models to understand the differential importance of many body effects depending on the core level ionized. The results that we have presented suggest that these many body effects are more important when the effective nuclear charges are more similar and less important when they are less similar. Thus, the many body effects will be larger for the $\mathrm{C}(1 \mathrm{~s})$ core ion of $\mathrm{CO}$, which is equivalent to $\mathrm{NO}^{+}$where the two nuclear charges differ by only one, and smaller for the $\mathrm{O}(1 \mathrm{~s})$ core of $\mathrm{CO}$, which is equivalent to $\mathrm{CF}^{+}$where the two nuclear charges differ by 3 . This correlation was first pointed out in Ref. [23]. We have also quantified the possible effects on core level BE shifts because the experimental data for the equivalent core molecules are at a different geometry than for the initial state geometry of the system before core ionization. We have shown that geometry changes of the magnitude expected between the ionized molecule and the equivalent core molecule can lead to changes in BEs of $\lesssim 0.5 \mathrm{eV}$. This suggests an uncertainty in the core level BE shifts obtained with the equivalent core model of order a few tenths of an $\mathrm{eV}$. To our knowledge, this quantification of the accuracy of the equivalent model has not been made before. We have demonstrated that while the equivalent core model can provide information about the $\mathrm{BE}$ shifts of the same atom at inequivalent positions in a system, it cannot be used to obtain information of the shifts of BEs between different atoms or to obtain absolute BEs. We have also shown that there are aspects of the XPS that cannot be treated with the equivalent core model yet these are aspects that can provide a great deal of information about the electronic structure of a 
system. These aspects concern the contributions of multiplets to the number and width of XPS features which is especially important for the XPS of open shell systems. The coupling which is not treated within the equivalent core model arises from the coupling of the open core shell with open valence shell electrons. Since the open core shell is not present in the equivalent core model systems, the multiplets do not arise naturally and would need to be added as an afterthought. It is likely that this core-valence coupling also needs to be taken into account for the intensities and the number of shake satellites. However, the most serious concern that we have identified is related to the distinction and separation of initial and final state contributions to XPS BE shifts.

We have stressed the importance of separating initial state and final state effects which is easily possible when rigorous core-ion WFs and DFT densities are used but is not possible within the current formulation of the equivalent core model. Indeed, this separation may not be possible within the equivalent core model because it may not be possible to define a frozen orbital, FO or KT, WF, see Eq. (1) or density as is possible with rigorous core hole treatments. We have considered, as an example of the importance of this separation, the decomposition of the origin of the surface core level shifts, SCLS, for two metal and two oxide surfaces. In all cases, we find that the dominant origin of the SCLS is from initial state contributions. It is particularly important that the dominance of the initial state contributions is found using both periodic and cluster models of the $\mathrm{CaO}$ (100) surface and using both WF and DFT models for the electronic structure. This consistency provides very strong support for the correctness of the decomposition into initial and final state contributions which is crucial to obtaining correct information from the XPS. It is even more important that the rigorous decomposition is different from the naïve conclusion that might be reached from equivalent core model analyses that the SCLS arises because of the different relaxation of bulk and surface core holes. Thus, while equivalent core-holes may provide a qualitative view of how screening may be different for different core holes, one must be cautious not to be misled and make incorrect assignments of the decomposition into initial and final contributions to BE shifts.

\section{Acknowledgements.}

PSB acknowledges support from the U.S. Department of Energy, Office of Science, Office of Basic Energy Sciences, Chemical Sciences, Geosciences, and Biosciences (CSGB) 
Division through the Geosciences program at Pacific Northwest National Laboratory. CS and FI have been supported by the Spanish Ministerio de Ciencia y Universidades (MICIUN) RTI2018095460-B-I00 through and Excellence María de Maeztu program MDM-2017-0767 grants and, in part, by Generalitat de Catalunya 2017SGR13 grant. 
Table I. Calculated and experimental $\mathrm{C}(1 \mathrm{~s})$ and $\mathrm{O}(1 \mathrm{~s}) \mathrm{BEs}$, in $\mathrm{eV}$, for $\mathrm{CO}$ using non-relativistic CAS WFs and including a relativistic correction, CAS-rel. The BE shift between $\mathrm{O}(1 \mathrm{~s})$ and $\mathrm{C}(1 \mathrm{~s}), \Delta \mathrm{BE}$ is also given. See text and Ref. [23] for details.

\begin{tabular}{|l|l|l|l|}
\hline & $\mathrm{BE}[\mathrm{C}(1 \mathrm{~s})]$ & $\mathrm{BE}[\mathrm{O}(1 \mathrm{~s})]$ & $\Delta \mathrm{BE}$ \\
\hline expt. & 296.2 & 542.6 & 246.3 \\
\hline CAS & 295.8 & 541.8 & 246.0 \\
\hline CAS-rel & 295.9 & 542.3 & 246.3 \\
\hline
\end{tabular}

Table II. Occupation numbers for the $\pi$ orbitals, $N(1 \pi)$ and $N(2 \pi)$, of the $C O$ and equivalent core CAS WFs for the ground, GS, C(1s) ions, and O(1s) ions. The shorthand, EQC, for the equivalent core model is used here and in Table III. The results for the all electron calculations for the GS and the $1 \mathrm{~s}$ ions are from Ref. [23] The ratio of the equivalent core, EQC-NO ${ }^{+}$and $\mathrm{EQC}-\mathrm{CF}^{+}$, $\mathrm{N}(2 \pi)$ to the rigorous core-hole values are given in parenthesis.

\begin{tabular}{|l|l|l|}
\hline & $\mathrm{N}(1 \pi)$ & $\mathrm{N}(2 \pi)$ \\
\hline $\mathrm{CO}-\mathrm{GS}$ & 3.908 & 0.092 \\
\hline $\mathrm{C}(1 \mathrm{~s})-\mathrm{CO}^{+}$ & 3.814 & 0.185 \\
\hline $\mathrm{O}(1 \mathrm{~s})-\mathrm{CO}^{+}$ & 3.946 & 0.054 \\
\hline EQC-NO & 3.832 & $0.168(0.90)$ \\
\hline EQC-CF $^{+}$ & 3.951 & $0.049(0.90)$ \\
\hline
\end{tabular}


Table III. Centers of charge, $<\mathrm{z}>$ in $\AA$, for the valence $\sigma$ and the $1 \pi$ and $2 \pi$ orbitals of the CAS WFs for the CO GS and $\mathrm{C}(1 \mathrm{~s})$ and $\mathrm{O}(1 \mathrm{~s})$ core-holes as well as for the equivalent core molecules, $\mathrm{NO}^{+}$and $\mathrm{CF}^{+}$. For the $\operatorname{sum}(\sigma), 1 \pi$, and $2 \pi<\mathrm{z}>$ of $\mathrm{NO}^{+}$and $\mathrm{CF}^{+}$, the ratio of the equivalent core to the core-hole $<\mathrm{z}>$ are given in parenthesis; see the caption to Table II and the text for details.

\begin{tabular}{|c|c|c|c|c|}
\hline \multicolumn{5}{|c|}{$\sigma$ Orbitals } \\
\hline & $3 \sigma$ & $4 \sigma$ & $5 \sigma$ & $\operatorname{sum}(\sigma)$ \\
\hline CO-GS & +0.26 & +0.60 & -0.85 & +0.02 \\
\hline $\mathrm{C}(1 \mathrm{~s})-\mathrm{CO}^{+}$ & +0.19 & +0.56 & -0.74 & +0.02 \\
\hline $\mathrm{O}(1 \mathrm{~s})-\mathrm{CO}^{+}$ & +0.29 & +0.65 & -0.86 & +0.14 \\
\hline $\mathrm{EQC}-\mathrm{NO}^{+}$ & +0.14 & +0.35 & -0.47 & $+0.05(2.2)$ \\
\hline $\mathrm{EQC}^{-\mathrm{CF}^{+}}$ & +0.35 & +0.56 & -0.84 & $+0.14(0.98)$ \\
\hline \multicolumn{5}{|c|}{$\pi$ Orbitals } \\
\hline & & $1 \pi$ & $2 \pi$ & \\
\hline CO-GS & & +0.27 & -010 . & \\
\hline $\mathrm{C}(1 \mathrm{~s})-\mathrm{CO}^{+}$ & & +0.14 & -0.09 & \\
\hline $\mathrm{O}(1 \mathrm{~s})-\mathrm{CO}^{+}$ & & +0.41 & +0.03 & \\
\hline${\mathrm{EQC}-\mathrm{NO}^{+}}^{-}$ & & $+0.15(1.06)$ & $-0.09(1.07)$ & \\
\hline $\mathrm{EQC}-\mathrm{CF}^{+}$ & & $+0.41(0.99)$ & $+0.02(0.62)$ & \\
\hline
\end{tabular}


Table IV. Changes, $\delta \mathrm{BE}[\mathrm{C}(1 \mathrm{~s})]$ and $\delta \mathrm{BE}[\mathrm{O}(1 \mathrm{~s})]$ in $\mathrm{eV}$, for changes in the $\mathrm{C}-\mathrm{O}$ distance, $\mathrm{d}(\mathrm{C}-\mathrm{O})$ from the BEs at $\mathrm{d}(\mathrm{C}-\mathrm{O})=\mathrm{r}_{\mathrm{e}}$. The BEs at $\mathrm{r}_{\mathrm{e}}$ are in Table I.

\begin{tabular}{|l|l|l|}
\hline $\mathrm{d}(\mathrm{C}-\mathrm{O})-\AA$ & $\delta \mathrm{BE}[\mathrm{C}(1 \mathrm{~s})]$ & $\delta \mathrm{BE}[\mathrm{O}(1 \mathrm{~s})]$ \\
\hline 1.032 & -0.74 & +0.28 \\
\hline 1.058 & -0.53 & +0.21 \\
\hline 1.085 & -0.32 & +0.13 \\
\hline 1.111 & -0.12 & +0.05 \\
\hline $1.128^{*}$ & 0.00 & 0.00 \\
\hline 1.138 & +0.07 & -0.03 \\
\hline 1.164 & +0.25 & -0.12 \\
\hline 1.191 & +0.41 & -0.20 \\
\hline
\end{tabular}

*This is the $\mathrm{CO}_{\mathrm{e}}$ 
Table V. Theoretical and experimental values, in eV, of the SCLS for $\mathrm{Cu}(100)$ and $\mathrm{Al}(100)$ and for $\mathrm{MgO}(100)$ and $\mathrm{CaO}(100)$ surfaces. The results are based on cluster models of the bulk and surfaces; for the CaO SCLS, periodic slab model DFT values are also given. Sources of the $\operatorname{SCLS}(\mathrm{KT}), \operatorname{SCLS}(\triangle \mathrm{SCF})$, and SCLS(Expt) are given as footnotes

\begin{tabular}{|l|l|l|l|l|}
\hline & & SCLS(KT) & SCLS( $\Delta$ SCF) & SCLS(Expt) \\
\hline $\mathrm{Cu}(100)^{\mathrm{a}}$ & $1 \mathrm{~s} \mathrm{BE}$ & -0.63 & -0.38 & -0.24 \\
\hline $\mathrm{Al}(100)^{\mathrm{a}}$ & $1 \mathrm{~s} \mathrm{BE}$ & +0.74 & +0.63 & ------ \\
\hline & $2 \mathrm{~s} \mathrm{BE}$ & +0.62 & ------ & ------ \\
\hline $\mathrm{MgO}(100)^{\mathrm{b}}$ & $2 \mathrm{p} \mathrm{BE}$ & +0.57 & ------ & +0.22 \\
\hline & $\mathrm{Mg} 2 \mathrm{p} \mathrm{BE}$ & +0.81 & +0.94 & +0.65 \\
\hline $\mathrm{CaO}(100)^{\mathrm{c}}-$ cluster & Ca 2p BE & +0.89 & +0.004 & unresolved \\
\hline & O 1s BE & +0.15 & +1.01 & +0.6 \\
\hline $\mathrm{CaO}(100)^{\mathrm{c}}-$ slab & Ca 2p BE & +0.58 & +0.05 & unresolved \\
\hline & O 1s BE & +0.19 & +0.78 & +0.6 \\
\hline
\end{tabular}

${ }^{a}$ For the SCLS values, see Refs. [72-73]

${ }^{\mathrm{b}}$ For the SCLS values, see Ref. [71]

${ }^{c}$ For the SCLS values, see Refs. [9, 70]; for the slab model SCLS(KT), see Ref. [76] 


\section{References}

1. W. L. Jolly and D. N. Hendrickson, "Thermodynamic interpretation of chemical shifts in coreelectron binding energies", J. Am. Chem. Soc. 92, 1863 (1970).

2. W. L. Jolly, "The estimation of core-electron binding-energy shifts using the concept of the equivalence of equally charged cores", in Proceedings of the international conference on electron spectroscopy, edited by D. A. Shirley (North-Holland, Amsterdam, Netherlands, 1972), p. 629.

3. J. W. Koepke and W. L. Jolly, "The core electron binding energies of oxygen difluoride", J. Electron Spectrosc. Relat. Phenom. 9, 413 (1976).

4. A. Rosengren and B. Johansson, "Surface heat of segregation from surface core-level bindingenergy shifts", Phys. Rev. B 23, 3852 (1981).

5. B. Johansson and N. Martensson, "Core-level binding-energy shifts for the metallic elements", Phys. Rev. B 21, 4427 (1980).

6. A. Rosengren and B. Johansson, "Calculated transition-metal surface core-level binding-energy shifts", Phys. Rev. B 22, 3706 (1980).

7. P. S. Bagus and H. F. Schaefer, III, "Localized and delocalized 1s hole states of the O2+ molecular ion", J. Chem. Phys. 56, 224 (1972).

8. F. A. Delesma et al., "A Chemical View on X-ray Photoelectron Spectroscopy: the ESCA Molecule and Surface-to-Bulk XPS Shifts", ChemPhysChem 19, 169 (2018).

9. P. S. Bagus et al., "Surface core level BE shifts for $\mathrm{CaO}(100)$ : insights into physical origins", Phys. Chem. Chem. Phys. 21, 25431 (2019).

10. P. S. Bagus, C. Sousa, and F. Illas, "Differential many-body effects for initial and core ionic states: impact on XPS spectra", Theor. Chem. Accounts 138, 61 (2019).

11. P. S. Bagus, E. S. Ilton, and C. J. Nelin, "Extracting Chemical Information From XPS Spectra: A Perspective", Catal. Lett. 148, 1785 (2018).

12. T. Aberg, "Theory of X-Ray Satellites", Phys. Rev. 156, 35 (1967).

13. J. C. Woicik et al., "Experimental assignment of many-electron excitations in the photoionization of NiO", Phys. Rev. B 97, 245142 (2018).

14. P. S. Bagus et al., "A New Mechanism For XPS Line Broadening: The 2p-XPS of Ti(IV)", The Journal of Physical Chemistry C 123, 7705 (2019). 
15. P. S. Bagus, C. R. Brundle, and E. S. Ilton, "A rigorous non-empirical theoretical analysis of the $2 p$ XPS of NiO: Is it necessary to invoke nonlocal screening?", Journal of Electroanalytical Chemistry, 114135 (2020).

16. P. S. Bagus, E. S. Ilton, and C. J. Nelin, "The Interpretation of XPS Spectra: Insights Into Materials Properties", Surf. Sci. Rep. 68, 273 (2013).

17. P. S. Bagus and F. Illas, "Orbitals Permit the Interpretation of Core-Level Spectroscopies in Terms of Chemistry", Catal. Lett. 50, 2457 (2020).

18. D. P. Chong, O. V. Gritsenko, and E. J. Baerends, "Interpretation of the Kohn-Sham orbital energies as approximate vertical ionization potentials", J. Chem. Phys. 116, 1760 (2002).

19. P. Hohenberg and W. Kohn, "Inhomogeneous Electron Gas", Phys. Rev. 136, B864 (1964).

20. W. Kohn and L. J. Sham, "Self-Consistent Equations Including Exchange and Correlation Effects", Phys. Rev. 140, A1133 (1965).

21. I. N. Levine, Quantum Chemistry (Prentice-Hall, Upper Saddle River, NJ, 2000).

22. T. Koopmans, Physica 1, 104 (1934).

23. P. S. Bagus, C. Sousa, and F. Illas, "Consequences of electron correlation for XPS binding energies: Representative case for C(1s) and O(1s) XPS of CO", J. Chem. Phys. 145, 144303 (2016).

24. R. S. Mulliken, J. chim. Phys. 46, 497 (1949).

25. P. A. Cox, "Ionization energies of open-shell molecules in the frozen orbital approximation", Mol. Phys. 30, 389 (1975).

26. N. P. Bellafont, F. Illas, and P. S. Bagus, "Validation of Koopmans' Theorem For Density Functional Theory Binding Energie", Phys. Chem. Chem. Phys. 17, 4015 (2015).

27. N. Pueyo Bellafont, P. S. Bagus, and F. Illas, "Prediction of core level binding energies in density functional theory: Rigorous definition of initial and final state contributions and implications on the physical meaning of Kohn-Sham energies", J. Chem. Phys. 142, 214102 (2015).

28. P. S. Bagus, F. Illas, and J. Casanovas, "The importance of $2 \mathrm{~s}$ bonding contributions for the core level binding energies in organic compounds", Chem. Phys. Lett. 272, 168 (1997).

29. P. S. Bagus, A. Wieckowski, and H. J. Freund, "Initial and final state contributions to bindingenergy shifts due to lattice strain: Validation of Auger parameter analyses", Chem. Phys. Lett. 420, 42 (2006). 
30. B. Richter et al., "Cluster core-level binding-energy shifts: The role of lattice strain", Phys. Rev. Lett. 93, 026805 (2004).

31. P. S. Bagus, "Self-Consistent-Field Wave Functions for Hole States of Some Ne-Like and Ar-Like Ions", Phys. Rev. 139, A619 (1965).

32. R. P. Gupta and S. K. Sen, "Calculation of multiplet structure of core p-vacancy levels. II", Phys. Rev. B 12, 15 (1975).

33. R. P. Gupta and S. K. Sen, "Calculation of multiplet structure of core p-vacancy levels", Phys. Rev. B 10, 71 (1974).

34. P. S. Bagus et al., "Analysis of the Fe 2p XPS for Hematite $\alpha$ Fe2O3: Consequences of Covalent Bonding and Orbital Splittings on Multiplet Splittings", J. Chem. Phys. 152, 014704 (2020).

35. B. D. Hermsmeier et al., "Energy dependence of the outer core-level multiplet structures in atomic Mn and Mn-containing compounds", Phys. Rev. B 48, 12425 (1993).

36. B. Hermsmeier et al., "Direct evidence from gas-phase atomic spectra for an unscreened intraatomic origin of outer-core multiplet splittings in solid manganese compounds", Phys. Rev. Lett. 61, 2592 (1988).

37. P. S. Bagus et al., "Multiplet splitting for the XPS of heavy elements: Dependence on oxidation state", Surf. Sci. 643, 142 (2016).

38. E. S. Ilton et al., "Quantifying small changes in uranium oxidation states using XPS of a shallow core level", Phys. Chem. Chem. Phys. 19, 30473 (2017).

39. P. S. Bagus, A. J. Freeman, and F. Sasaki, "Prediction of new multiplet structure in photoemission experiments", Phys. Rev. Lett. 30, 850 (1973).

40. P. S. Bagus, R. Broer, and E. S. Ilton, "Atomic near-degeneracy for photoemission: Generality of $4 \mathrm{f}$ excitations", J. Electron Spectrosc. Relat. Phenom. 165, 46 (2008).

41. P. S. Bagus, R. Broer, and E. S. Ilton, "A new near degeneracy effect for photoemission in transition metals", Chem. Phys. Lett. 394, 150 (2004).

42. C. S. Fadley, "Basic Concepts of X-Ray Photoelectron Spectroscopy", in Electron Spectroscopy: Theory, Techniques and Applications, edited by C. R. Brundle and A. D. Baker (Academic Press, 1978), Vol. 2, p. 2.

43. P. S. Bagus and J. V. Mallow, "An atomic model for the spin-polarized photoemission spectra of Fe", Chem. Phys. Lett. 228, 695 (1994). 
44. B. Brena, S. Carniato, and Y. Luo, "Functional and basis set dependence of K-edge shake-up spectra of molecules", J. Chem. Phys. 122, 184316 (2005).

45. B. Brena et al., "Equivalent core-hole time-dependent density functional theory calculations of carbon \$1s\$ shake-up states of phthalocyanine", Phys. Rev. B 70, 195214 (2004).

46. W. Hua, G. Tian, and Y. Luo, "Theoretical assessment of vibrationally resolved C1s X-ray photoelectron spectra of simple cyclic molecules", Phys. Chem. Chem. Phys. (2020).

47. J.-R. Zhang et al., "Accurate K-edge X-ray photoelectron and absorption spectra of g-C3N4 nanosheets by first-principles simulations and reinterpretations", Phys. Chem. Chem. Phys. 21, 22819 (2019).

48. S. Lunell et al., "A theoretical and experimental study of the carbon 1s shake-up structure of benzene", Chem. Phys. Lett. 54, 420 (1978).

49. B. Sjögren, "A theoretical study of the shake-up intensity of benzene", J. Chem. Phys. 96, 8338 (1992).

50. K. Weiss, P. S. Bagus, and C. Woll, "Rydberg transitions in X-ray absorption spectroscopy of alkanes: The importance of matrix effects", J. Chem. Phys. 111, 6834 (1999).

51. C. Wöll, K. Weiss, and P. S. Bagus, "Saturated hydrocarbons on a Cu surface: a new type of chemical interaction?", Chem. Phys. Lett. 332, 553 (2000).

52. R. Manne and T. Åberg, Chem. Phys. Lett. 7, 282 (1970).

53. L. Yang and H. Ågren, "Can substituted benzenes be used as building blocks in core electron shake-up spectra?", Chem. Phys. 222, 139 (1997).

54. E. E. Rennie et al., "A comprehensive photoabsorption, photoionization, and shake-up excitation study of the C 1s cross section of benzene", J. Chem. Phys. 113, 7362 (2000).

55. N. Martensson, S. Svensson, and U. Gelius, "Single and double shake-up processes in the Ne $1 \mathrm{~s}$ photoelectron spectrum", Journal of Physics B: Atomic and Molecular Physics 20, 6243 (1987).

56. B. O. Roos, P. R. Taylor, and P. E. M. Siegbahn, "A complete active space SCF method (CASSCF) using a density matrix formulated super-Cl approach", Chem. Phys. 48, 157 (1980).

57. J. Cioslowski, "Density-driven self-consistent-field method: density-constrained correlation energies in the helium series", Phys. Rev. A 43, 1223 (1991). 
58. E. Valderrama, E. V. Ludena, and J. Hinze, "Assessment of dynamical and nondynamical correlation energy components for the beryllium-atom isoelectronic sequence", J. Chem. Phys. 110, 2343 (1999).

59. K. P. Huber and G. Herzberg, Molecular Spectra and Molecular Structure Vol. 4, Constants of Diatomic Molecules (Van Nostrand, New York, 1979).

60. S. R. Smith and T. Darrah Thomas, "Core ionization potentials in carbon monoxide", J. Electron Spectrosc. Relat. Phenom. 8, 45 (1976).

61. P. O. Löwdin, "Quantum Theory of Many-Particle Systems. I. Physical Interpertations by Means of Density Matrices, Natural Spin-Orbitals, and Convergence Problems in the Method of Configurational Interaction", Phys. Rev. 97, 1474 (1955).

62. J. C. Slater, Phys. Rev. 36, 57 (1930).

63. P. S. Bagus and E. K. Viinikka, "Origin of satellite structure in the valence X-ray photoelectron spectrum of CO: A theoretical study", Phys. Rev. A 15, 1486 (1977).

64. C. J. Nelin et al., "Analysis of the Broadening of X-ray Photoelectron Spectroscopy Peaks for lonic Crystals", Angew. Chem. Int. Ed. 50, 10174 (2011).

65. U. Gelius et al., "Vibrational and lifetime line broadenings in ESCA", Chem. Phys. Lett. 28, 1 (1974).

66. M. Iwan and C. Kunz, "Investigation of the phonon broadening of core levels in $\mathrm{NaCl}$ ", Phys. Lett. A 60, 345 (1977).

67. H. P. Steinruck et al., "A detailed analysis of vibrational excitations in x-ray photoelectron spectra of adsorbed small hydrocarbons", J. Chem. Phys. 125 (2006).

68. P. S. Bagus, C. J. Nelin, and E. S. Ilton, "Theoretical modeling of the uranium $4 \mathrm{f} X P S$ for $U(V I)$ and U(IV) oxides", J. Chem. Phys. 139, 244704 (2013).

69. W. F. Egelhoff, "Core-Level Binding-Energy Shifts at Surfaces and In Solids", Surf. Sci. Rep. 6, 253 (1987).

70. P. S. Bagus et al., "Revisiting surface core-level shifts for ionic compounds", Phys. Rev. B 100, 115419 (2019).

71. C. J. Nelin et al., "Surface core-level binding energy shifts for $\mathrm{MgO}(100)$ ", Phys. Chem. Chem. Phys. 16, 21953 (2014). 
72. P. S. Bagus et al., "Mechanisms responsible for the shifts of core-level binding energies between surface and bulk atoms of metals", Surf. Sci. Rep. 19, 265 (1993).

73. P. S. Bagus, G. Pacchioni, and F. Parmigiani, "Surface core-level spectroscopy of $\mathrm{Cu}(100)$ and Al(100)", Phys. Rev. B 43, 5172 (1991).

74. R. W. G. Wyckoff, Crystal Structures (Wiley, New York, 1963).

75. H. M. Evjen, "On The Stability of Certain Heteropolar Crystals", Phys. Rev. 39, 675 (1932).

76. X. Zhao and S. V. Levchenko, private communication). 